\section{Visual outcomes in patients with zonular dialysis following cataract surgery}

S Trikha ${ }^{1,2}$, S Agrawal ${ }^{1}$, S-E Saffari ${ }^{2,3}$, R Jayaswal ${ }^{1}$ and YF Yang ${ }^{1}$

\begin{abstract}
Purpose To evaluate the visual outcomes of patients with zonular dialysis following cataract surgery.

Patients and methods Medical records of all patients with documented zonular dialysis, either pre- or peri-operatively, undergoing cataract surgery between 2004-2010 at Queen Alexandra Hospital, Portsmouth, were retrospectively reviewed. Baseline demographics and biometry were analysed, and ocular co-morbidities documented. Intraoperative complications and the use of a capsular tension ring (CTR) were identified. Early and late best-corrected visual acuity (BCVA) post surgery were determined using LogMar values. Univariate and multivariate linear regression analysis was performed to determine associations with BCVA post surgery, and further subgroup analysis performed in groups determined by CTR use.
\end{abstract}

Results The records of 22312 consecutive eyes undergoing cataract surgery were reviewed. The incidence of zonular dialysis was $0.50 \%$ (111 eyes). A CTR was inserted in 46 eyes. Using a multivariate linear regression model, better initial pre-operative BCVA $(P=0.019)$, the use of a CTR $(P=0.014)$, and the absence of vitreous loss during surgery $(P=0.008, \beta$ 0.45) were associated with improved early postoperative BCVA (mean follow-up 6.6 weeks). Better medium-term postoperative BCVA was significantly associated with preoperative BCVA $(P=0.002)$ and the use of a CTR during surgery $(P=0.004, \beta-0.41)$.

Conclusions The overall incidence of zonular dialysis is low. CTR use intraoperatively suggests improved early and medium-term BCVA and should be considered in all cases of zonular dialysis.

Eye (2016) 30, 1331-1335; doi:10.1038/eye.2016.108; published online 10 June 2016

\section{Introduction}

Zonular dialysis, whether inherited, acquired or iatrogenic, often presents unique challenges to the cataract surgeon. While the presence of zonular dialysis has commonly been reported in pseudoexfoliation syndrome (PXF) or trauma, studies reporting the incidence, and specifically visual outcomes, of zonular dialysis detected at cataract surgery are limited. ${ }^{1}$ The UK CND audit of 55297 patients identified a zonular dialysis rate of $0.46 \% .^{2}$

Zonular dialysis has been associated with a variety of adverse outcomes including early or late in-the-bag IOL dislocation, and progressive asymmetrical capsular contraction resulting in IOL decentration. ${ }^{3,4}$ Capsule tension rings (CTRs) have been reported to compensate for weakened or damaged zonules in a number of ways, acting as a supportive device both intraand postoperatively, but have advantages and disadvantages. ${ }^{4-6}$ The CTR can redistribute mechanical forces induced by nuclear sculpting or IOL insertion. ${ }^{4}$ Similarly, the CTR induces centripetal pressure, reducing capsular folds, preventing forward movement of the posterior capsule, and thereby countering progressive contractile capsular forces post-operatively. ${ }^{4,7}$ In contrast, compression is often required to implant the CTR, which itself may introduce further zonular damage. ${ }^{4,8}$ Furthermore, CTR may prevent full cortical aspiration by trapping the cortex within the capsular fornix. ${ }^{4}$ Despite these findings, medium to long-term outcome data in the use of CTR are lacking.

The aims of our study were to report the incidence and visual outcomes of patients with zonular dialysis undergoing cataract surgery. Further subgroup analysis compared visual outcomes in the CTR insertion and non-insertion groups.
${ }^{1}$ Department of Ophthalmology, Queen Alexandra Hospital, Cosham, Portsmouth, Hampshire, UK

${ }^{2}$ Singapore Eye Research Institute, Singapore National Eye Centre, Singapore

${ }^{3}$ Duke-NUS Graduate Medical School, Singapore

Correspondence: YF Yang, Department of Ophthalmology, Queen Alexandra Hospital, Cosham, Portsmouth PO6 3LY, Hampshire, UK Phone: +44 (0)23 9228 6000; Fax: +44 (0)23 92286440 . E-Mail: yitfung.yang@ porthosp.nhs.uk

Received: 11 September 2015 Accepted in revised form: 15 April 2016 Published online: 10 June 2016 


\section{Materials and methods}

Electronic medical records (Medisoft Ophthalmology, Medisoft Limited, Leeds, UK) of all consecutive patients with documented zonular dialysis during cataract surgery, from June 2004 to November 2010, were retrospectively reviewed by a senior ophthalmic surgeon.

All patients underwent a complete slit-lamp examination, including autorefraction (Nidek ARK, Fremont, CA, USA), applanation tonometry (Goldmann, Haag-Streit, Switzerland), and dilated fundus examination, both pre- and postoperatively. Baseline demographics such as age and gender were documented from the records, along with pre-existing ocular co-morbidities. Pre-operative biometry (IOL Master, Carl-Zeiss Meditec, Dublin, USA) and intended post-operative spherical equivalent were also noted. Data on the pre-operative pupil size, subjectively measured by the grade of surgeon performing the procedure, the use of a CTR (Morcher, FCI Ophthalmics, Pembroke, MA, USA), and intraoperative complications were also collected. Postoperatively, early and last BCVA were documented from follow-up.

\section{Statistical analysis}

Data were analysed using SAS version 9.3 for Windows (SAS, Inc., Cary, NC, USA). Baseline demographic and clinical features were reported for the 111 eyes, with the diagnosis of zonular dialysis done before or during surgery. In multivariate linear regression analysis of the continuous outcome of early postoperative BCVA (LogMAR), we excluded all clinical features or baseline demographics that did not achieve the $P<0.15$ significance level in the univariate analysis. Variables included in the univariate analysis were most recent preoperative BCVA (LogMAR), age, and gender, including diagnoses of trauma and/or glaucoma, diabeteic retinopathy, uveitis with/without posterior synechiae, phacodonesis, and PXF. Further variables included were whether a pre-procedure YAG laser peripheral iridotomy had been performed, the grade of operating surgeon, pupil size pre-procedure, CTR (use/no use), vitreous loss, PCIOL insertion, keratometry, axial length, and anterior chamber washout. A multivariate linear regression analysis was used to assess the association between the late postoperative BCVA (as a continuous outcome, LogMAR unit, in patients with more than 6 months follow-up) and the clinical features or baseline demographics, where the adjustment was for the variables with a $P<0.15$ significance level in the univariate analysis.

The absolute differences between the actual and predicted values of spherical equivalent were compared
Table 1 Summary of cases with zonular dialysis detected intra-operatively

\begin{tabular}{lcc}
\hline Variable & $\mathrm{n}$ & Count $(\%) /$ mean $\pm S D$ \\
\hline Age & 111 & $78.8 \pm 8.9$ \\
Gender (male) & 111 & $51(45.9 \%)$ \\
CTR (used) & 110 & $46(41.8 \%)$ \\
Vitreous loss & 110 & $22(20 \%)$ \\
Glaucoma & 111 & $21(18.9 \%)$ \\
Uveitis/synechiae & 111 & $5(4.5 \%)$ \\
\hline
\end{tabular}

based on CTR (used versus did not use) using a Mann-Whitney $U$ test. Statistical significance was set at $P \leq 0.05$.

\section{Results}

A total of 22312 consecutive phacoemulsification surgical procedures were carried out during the study period. Of these, in $0.50 \%$ (111 eyes) the diagnosis of zonular dialysis was done during surgery (3 were confirmed preoperatively), with or without vitreous loss (VL). Fifty-one patients were male and 60 female. The mean age of patients, at surgery, was 78.9 years (SD 8.9 years). In 22 cases $(20 \%)$ zonular dialysis was associated with VL (Table 1). In the case of VL, 4 eyes had an AC-IOL inserted, 11 eyes a sulcus IOL, 6 a PC-IOL, and 1 eye was left aphakic. ZD incidence CTR use ranged from 4 cases in 2004 to 24 cases in 2005, 17 in 2006, 16 in 2007, 18 in 2008, 18 in 2009, and 14 in 2010. Data on CTR use per individual surgical firm were not available. Data on CTR use by individual surgical firm were not available for analysis and interpretation, given the general waiting list pool that patients were selected for.

Early postoperative BCVA (110 patients, mean 6.6 weeks, SD 8.6 weeks) was significantly associated with preoperative $\operatorname{BCVA}(\beta 0.28, P=0.019)$, positively associated with the use of a CTR $(\beta-0.34, P=0.013)$, and negatively associated with the presence of $\mathrm{VL}$ during surgery $(\beta 0.45, P=0.008)$ (Table 2$)$.

Medium-term follow-up (greater than 6 months) BCVA was obtained for 70 patients (mean 34.2 months, SD 23.4 months). Using a multivariate logistic regression model, better late postoperative BCVA was found significantly associated with preoperative BCVA and the intra-operative use of a CTR $(\beta-0.40, P=0.004)$ (Table 3$)$. Of note, one case of late in-the-bag IOL dislocation was detected in a case without CTR use. No significant difference in the actual versus predicted spherical equivalent was noted in the use of a CTR $(P=0.834)$ (Table 4). 
Table 2 Univariate and multivariate analysis of associations with early post-operative BCVA

\begin{tabular}{|c|c|c|c|c|}
\hline \multirow{2}{*}{ Parameter } & \multicolumn{2}{|c|}{ Univariate } & \multicolumn{2}{|c|}{ Multivariate } \\
\hline & Est. $(95 \%$ CI) & P value & Adj est. $(95 \%$ CI) & $\mathrm{P}$ value \\
\hline Pre-op BCVA & $0.44(0.19,0.68)$ & 0.001 & $0.28(0.05,0.51)$ & 0.019 \\
\hline Age (years) & $0(-0.02,0.02)$ & 0.795 & & \\
\hline Gender & $0.12(-0.21,0.44)$ & 0.470 & & \\
\hline CTR use $(\mathrm{Y} / \mathrm{N})$ & $-0.47(-0.78,-0.17)$ & 0.003 & $-0.34(-0.6,-0.07)$ & 0.013 \\
\hline Vitreous loss $(\mathrm{Y} / \mathrm{N})$ & $0.52(0.16,0.87)$ & 0.005 & $0.45(0.12,0.78)$ & 0.008 \\
\hline Trauma (Y/N) & $0.82(-0.13,1.76)$ & 0.088 & $0.72(-0.07,1,51)$ & 0.075 \\
\hline Glaucoma $(\mathrm{Y} / \mathrm{N})$ & $-0.21(-0.61,0.19)$ & 0.289 & & \\
\hline Surgeon grade (Cons/Reg) & $-0.19(-0.53,0.16)$ & 0.290 & & \\
\hline YAG laser PI (Y/N) & $-0.29(-0.91,0.33)$ & 0.360 & & \\
\hline Uveitis/synechiae (Y/N) & $-0.19(-0.88,0.5)$ & 0.588 & & \\
\hline $\mathrm{PXF} /$ phacodonesis $(\mathrm{Y} / \mathrm{N})$ & $0.13(-0.35,0.6)$ & 0.601 & & \\
\hline PCIOL insertion $(\mathrm{Y} / \mathrm{N})$ & $-0.21(-1.18,0.75)$ & 0.660 & & \\
\hline $\mathrm{AC}$ wash $(\mathrm{Y} / \mathrm{N})$ & $-0.01(-0.12,0.09)$ & 0.771 & & \\
\hline Axial length & $0.01(-0.12,0.13)$ & 0.929 & & \\
\hline Keratometry K2 & $-0.18(-1.53,1.17)$ & 0.792 & & \\
\hline Keratometry $\mathrm{K} 1$ & $0.01(-0.09,0.11)$ & 0.889 & & \\
\hline Pupil size (S/L) & $0.31(-0.2,0.82)$ & 0.235 & & \\
\hline Pupil size (M/L) & $-0.06(-0.76,0.64)$ & 0.860 & & \\
\hline Diabetic ret $(\mathrm{Y} / \mathrm{N})$ & $0.03(-0.93,1)$ & 0.944 & & \\
\hline
\end{tabular}

Table 3 Univariate and multivariate analysis of parameters associated with medium-term post-operative BCVA

\begin{tabular}{|c|c|c|c|c|}
\hline \multirow{2}{*}{ Parameter } & \multicolumn{2}{|c|}{ Univariate } & \multicolumn{2}{|c|}{ Multivariate } \\
\hline & Est $(95 \%$ CI $)$ & P value & Adj est $(95 \%$ CI $)$ & $\mathrm{P}$ value \\
\hline Pre-op BCVA & $0.43(0.19,0.68)$ & 0.001 & $0.36(0.13,0.59)$ & 0.002 \\
\hline Age (years) & $0.01(0,0.03)$ & 0.117 & $0.01(0,0.03)$ & 0.098 \\
\hline CTR use $(\mathrm{Y} / \mathrm{N})$ & $-0.47(-0.76,-0.19)$ & 0.002 & $-0.4(-0.68,-0.13)$ & 0.004 \\
\hline Gender $(\mathrm{F} / \mathrm{M})$ & $-0.01(-0.32,0.29)$ & 0.922 & & \\
\hline YAG laser PI $(\mathrm{Y} / \mathrm{N})$ & $-0.38(-0.97,0.21)$ & 0.200 & & \\
\hline Axial length & $-0.07(-0.22,0.08)$ & 0.379 & & \\
\hline Keratometry K2 & $-0.02(-0.12,0.09)$ & 0.763 & & \\
\hline Keratometry K1 & $0.01(-0.1,0.11)$ & 0.873 & & \\
\hline Glaucoma $(\mathrm{Y} / \mathrm{N})$ & $0.14(-0.21,0.5)$ & 0.426 & & \\
\hline Surgeon grade (Cons/Reg) & $-0.09(-0.43,0.25)$ & 0.600 & & \\
\hline Trauma $(\mathrm{Y} / \mathrm{N})$ & $-0.15(-0.74,0.44)$ & 0.610 & & \\
\hline Pupil size (S/L) & $0.39(-0.06,0.84)$ & 0.089 & & \\
\hline Pupil size (M/L) & $-0.16(-0.9,0.59)$ & 0.678 & & \\
\hline PCIOL insertion $(\mathrm{Y} / \mathrm{N})$ & $0.15(-0.6,0.91)$ & 0.686 & & \\
\hline Uveitis/synechiae (Y/N) & $-0.01(-0.77,0.74)$ & 0.971 & & \\
\hline $\mathrm{PXF} /$ phacodonesis $(\mathrm{Y} / \mathrm{N})$ & $0.1(-0.41,0.61)$ & 0.688 & & \\
\hline $\mathrm{AC}$ wash $(\mathrm{Y} / \mathrm{N})$ & $-0.26(-1.54,1.03)$ & 0.691 & & \\
\hline Diabetic ret $(\mathrm{Y} / \mathrm{N})$ & $0.09(-0.42,0.59)$ & 0.734 & & \\
\hline Vitreous loss $(\mathrm{Y} / \mathrm{N})$ & $0.06(-0.3,0.42)$ & 0.757 & & \\
\hline
\end{tabular}

Table 4 Comparison of the absolute differences for actual and predicted values of spherical equivalent based on CTR $(\mathrm{Y} / \mathrm{N})$

\begin{tabular}{lccc}
\hline CTR & Used & Did not use & P value \\
\hline Mean \pm SD & $0.71 \pm 0.63$ & $0.70 \pm 0.60$ & 0.834 \\
$\mathrm{n}$ & 42 & 48 & \\
\hline
\end{tabular}

${ }^{a}$ Mann-Whitney U test for non-parametric distribution.

\section{Discussion}

This study reports improved BCVA postoperatively, over the medium term, in patients receiving a CTR for zonular dialysis during phacoemulsifcation surgery in a large consecutive cohort of patients. This compares favourably to a study by Wang et $a l^{9}$ that illustrated that CTR use for zonular dialysis was associated with an improved 
postoperative BCVA in $72.6 \%$ of cases. Furthermore, the overall incidence of zonular dialysis without VL in our cohort ( 89 cases, $0.4 \%$ ) is similar to the data from the UK CND EPR audit of $0.46 \% .^{2}$ In addition, the overall use of a CTR in our study $(0.5 \%)$ compares favourably to CTR use in a similar study by Tribus et $a l^{10}$ (69 out of 9528 consecutive cataract procedures, $0.7 \%$ ).

Our study also showed no statistically significant difference in the spherical equivalent outcome with or without the use of a CTR, in agreement with a study by Boomer and Jackson ${ }^{11}$ that found no consistent association between CTR use and refractive outcome. Another study by Schild et al, ${ }^{12}$ looking at refractive outcomes in myopic eyes undergoing phacoemulsification with and without CTR use, suggested that the IOL power calculation does not have to be adjusted when a CTR is used.

Reports of visual outcomes following zonular dialysis have hitherto been limited by follow-up period, sample size, and no adjustment for pre-operative BCVA. ${ }^{13} \mathrm{~A}$ prospective study of 21 eyes from 19 patients, with documented zonular dialysis and CTR use, showed that 15 eyes $(74.42 \%$ ) had a postoperative BCVA of $6 / 12$ or better. ${ }^{13}$ Similarly, a study by Ionides et al ${ }^{14}$ assessed BCVA in 17 patients with zonular dialysis, and found an acuity of $6 / 12$ or better in $80 \%$ of patients with no previous ocular co-morbidity, and in $42.9 \%$ of patients with ocular co-morbidity.

The use of a CTR has potential advantages, including the symmetrical distribution of capsular tension, thereby ensuring reduced capsular contraction and IOL dislocation - a phenomenon first described by Davison ${ }^{15}$ in 1993. Furthermore, IOL dislocation has been documented following irregular capsulorrhexis creation in the presence of zonular dialysis. ${ }^{3,16}$ However, some evidence exists suggesting that CTR insertion will not exclusively prevent IOL dislocation. A total of 21 out of $84(27 \%)$ in a study by Jakobsson et al, and 8 out of $45(18 \%)$ in a study by Lorente et al had IOL dislocation following CTR insertion. Both of these studies had high frequencies of eyes with PPXF syndrome (60\% and $66.7 \%$, respectively). ${ }^{16,17}$ Although this is likely to be a major risk factor for late IOL dislocation, the possibility of iatrogenic damage during, and mis-timing of CTR insertion, cannot be discounted. ${ }^{8}$ In comparison, our study revealed that 8 patients with zonular dialysis had PXF, of whom $4(50 \%)$ underwent CTR insertion during surgery. The one case of documented late-in-the-bag IOL dislocation was in a patient with PXF and no CTR insertion. The low detection rate may therefore be a true reflection of the incidence of IOL dislocation, or under-reporting due to the medium follow-up period in our study. Indeed, Jehan et $\mathrm{al}^{18}$ reported 8 cases of late in-the-bag IOL subluxation/ dislocation at a mean time point of 7 years post surgery, and another study reported 86 cases at a mean of 8.5 years. ${ }^{19}$ In both studies no CTR was inserted, suggesting that CTR use does provide some protection in IOL dislocation. However, as some causes of zonular weakness may be progressive, longer follow-up may be required. With a small PXF cohort, our study does not prove the protective effect of CTR on IOL decentration or dislocation, as suggested by other studies. ${ }^{16}$ One study reported earlier late in-the-bag IOL dislocation in patients with CTR insertion than in those without, although selection bias may have meant that CTRs were inserted in the more severe zonular dialysis. ${ }^{17}$

This study has a number of limitations. While being retrospective in nature, the case capture period is 6 years and incidence of zonular dialysis was identified from 22312 cases, resulting in a large consecutive cohort of eyes with zonular dialysis. Although this study utilised electronic records for pre- and perioperative cataract surgery data, this has the advantage of being documented in a standardised format, therefore minimising bias or data loss. Paper-based records were then utilised for later postoperative review and final visual outcome.

Preoperative zonular dialysis was detected through clinician judgement rather than subjective measures, including ultrasound biomicroscopy (UBM), and surgical judgement was used intra-operatively to determine the use of a CTR. The exact technique of CTR insertion was also not documented. These variations are, however, likely to be representative of true clinical practice. Selection bias is also present as medium-term follow-up data are likely to be obtained by patients with ocular comorbidities, with others potentially discharged from clinic. Furthermore, the sample size is small due to the low incidence of zonular dialysis, and hence may be underpowered to reveal other significant associations. Although the follow-up period was nearly 3 years, this may be insufficient to identify all cases of late IOL dislocation.

In summary, our study suggests that improved early and medium-term visual outcomes can be obtained with the use of CTR in all cases of zonular dialysis detected during phacoemulsification surgery. The incidence of zonular dialysis itself is rare, as demonstrated by this study of a large cohort of patients undergoing cataract surgery in a UK district general hospital, and is reflective of likely clinical practice. Although the presence of selection bias limits long-term outcome data, outside of randomised, long-term trials, CTR use appears to be beneficial and should be strongly considered in all cases of zonular dialysis. 


\section{Summary}

What was known before

- Zonular dialysis is a relatively rare complication of cataract surgery.

- Small case series, with short follow-up, have reported variable visual acuity outcomes.

What this study adds

- The use of a capsule tension ring (CTR) is associated with improved early and medium-term visual acuity outcomes.

- CTR use should be considered in all cases of zonular dialysis.

\section{Conflict of interest}

The authors declare no conflict of interest.

\section{References}

1 Dwivedi NR, Dubey AK, Shankar PR. Intraoperative and Immediate Postoperative Outcomes of Cataract Surgery using Phacoemulsification in Eyes with and without Pseudoexfoliation Syndrome. J Clin Diagn Res 2014; 8(12): VC01-VC05.

2 Narendran N, Jaycock P, Johnston RL, Taylor H, Adams M, Tole DM et al. The Cataract National Dataset electronic multicentre audit of 55,567 operations: risk stratification for posterior capsule rupture and vitreous loss. Eye (Lond) 2009; 23(1): 31-37.

3 Gimbel HV. Role of capsular tension rings in preventing capsular contraction. J Cataract Refract Surg 2000; 26: 791-792.

4 Werner L, Zaugg B, Neuhann T, Burrow M, Tetz M. In the Bag Capsular Tension Ring and intraocular lens Subluxation or Dislocation. Ophthalmology 2012; 119: 266-271.

5 Nagamoto T, Bissen-Miyajima H. A ring to support the cap- sular bag after continuous curvilinear capsulorhexis. J Cataract Refract Surg 1994; 20: 417-420.

6 Weber $\mathrm{CH}$, Cionni RJ. All about capsular tension rings. Curr Opin Ophthalmol. 2015; 26(1): 10-15.

7 Gimbel HV, Condon GP, Kohnen T, Olson RJ, Halkiadakis I. Late in-the-bag intraocular lens dislocation: incidence, prevention, and management. J Cataract Refract Surg 2005; 31(11): 2193-2204.
8 Ahmed II, Cionni RJ, Kranemann C, Crandall AS. Optimal timing of capsular tension ring implantation: Miyake-Apple video analysis. J Cataract Refract Surg 2005; 31: 1809-1813.

9 Wang BZ, Chan E, Vajpayee R. A retrospective study of the indications and outcomes of capsular tension ring insertion during cataract surgery at a tertiary teaching hospital. Clin Ophthalmol 2013; 7: 567-572.

10 Tribus C, Alge CS, Hantoglou C, Lackerbauer C, Kampik A, Mueller A et al. Indication and clinical outcome of capsular tension ring (CTR) implantation: a review of 9528 cataract surgeries. Clin Ophthalmol 2007; 1(1): 65-69.

11 Boomer JA, Jackson DW. Effect of the Morcher capsular tension ring on refractive outcome. J Cataract Refract Surg. 2006; 32(7): 1180-1183.

12 Schild AM, Rosentreter A, Hellmich M, Lappas A, Dinslage S, Dietlein TS. Effect of a capsular tension ring on refractive outcomes in eyes with high myopia. J Cataract Refract Surg 2010; 36: 2087-2093.

13 Jacob S, Agarwal A, Agarwal A, Agarwal S, Patel N, Lal V. Efficacy of a capsular tension ring for phacoemulsification in eyes with zonular dialysis. J Cataract Refract Surg. 2003; 29(2): 315-321.

14 Ionides A, Minassian D, Tuft S. Visual outcome following posterior capsule rupture during cataract surgery. Br J Ophthalmol. 2001; 85(2): 222-224.

15 Davison JA. Capsule contraction syndrome. J Cataract Refract Surg. 1993; 19: 582-589.

16 Jakobsson G, Zetterberg M, Lundström M, Steveni U, Grenmark R, Sundelin K. Late dislocation of in-the-bag and out-of-the bag intraocular lenses: ocular and surgical characteristics and time to lens repositioning. J Cataract Refract Surg. 2010; 36(10): 1637-1644.

17 Lorente R, de Rojas V, Vazquez de Parga P, Moreno C, Landaluce ML, Dominguez $\mathrm{R}$ et al. Management of late spontaneous in-the-bag intraocular lens dislocation: retrospective analysis of 45 cases. J Cataract Refract Surg 2010; 36: 1270-1282.

18 Jehan FS, Mamalis N, Crandall AS. Spontaneous late dislocation of intraocular lens within the capsular bag in pseudo-exfoliation patients. Ophthalmology 2001; 108: 1727-1731.

19 Davis D, Brubaker J, Espandar L, Stringham J, Crandall A, Werner L et al. Late in-the-bag spontaneous intraocular lens dislocation: evaluation of 86 consecutive cases. Ophthalmology 2009; 116: 664-670. 